\title{
Extraction of Next-to-Next-to-Leading-Order Parton Distribution Functions from Lattice QCD Calculations
}

\author{
Zheng-Yang Li, ${ }^{1, *}$ Yan-Qing Ma®, ${ }^{1,2,3, \dagger}$ and Jian-Wei Qiu ${ }^{4, *}$ \\ ${ }^{1}$ School of Physics and State Key Laboratory of Nuclear Physics and Technology, Peking University, Beijing 100871, China \\ ${ }^{2}$ Center for High Energy physics, Peking University, Beijing 100871, China \\ ${ }^{3}$ Collaborative Innovation Center of Quantum Matter, Beijing 100871, China \\ ${ }^{4}$ Theory Center, Jefferson Lab, 12000 Jefferson Avenue, Newport News, Virginia 23606, USA
}

(Received 2 July 2020; revised 5 October 2020; accepted 29 November 2020; published 19 February 2021)

\begin{abstract}
We present for the first time complete next-to-next-to-leading-order coefficient functions to match flavor nonsinglet quark correlation functions in position space, which are calculable in lattice QCD, to parton distribution functions (PDFs). Using PDFs extracted from experimental data and our calculated matching coefficients, we predict valence-quark correlation functions that can be confronted by lattice QCD calculations. The uncertainty of our predictions is greatly reduced with higher order matching coefficients. By performing Fourier transformation, we also obtain matching coefficients for corresponding quasi-PDFs and pseudo-PDFs. Our method of calculations can be readily generalized to evaluate the matching coefficients for sea-quark and gluon correlation functions, making the program to extract partonic structure of hadrons from lattice QCD calculations comparable with and complementary to that from experimental measurements.
\end{abstract}

DOI: 10.1103/PhysRevLett.126.072001

Introduction.-Parton distribution functions (PDFs) encode the important nonperturbative information of strong interactions, and they are crucial for understanding all phenomena at the Large Hadron Collider (LHC) [1]. In terms of QCD factorization [2], a typical hadronic cross section with a large momentum transfer $Q$ and collision energy $\sqrt{S}$ at the LHC can be factorized as

$$
\begin{aligned}
d \sigma_{h h^{\prime}}\left(Q^{2}, S\right)= & \sum_{i, j} f_{i / h}\left(x, \mu^{2}\right) \otimes f_{j / h^{\prime}}\left(x^{\prime}, \mu^{2}\right) \\
& \otimes d \hat{\sigma}_{i j}\left(x, x^{\prime}, \mu^{2}, Q^{2}, S\right)+O\left(\Lambda_{\mathrm{QCD}}^{2} / Q^{2}\right),
\end{aligned}
$$

where $i, j=q, \bar{q}, g$ represents parton flavor; $f_{i / h}\left(x, \mu^{2}\right)$ is the PDF as a probability distribution to find an active parton of flavor $i$ inside a colliding hadron $h$ with the parton carrying the hadron's momentum fraction $x$, probed at a factorization scale $\mu \sim O(Q) ; d \hat{\sigma}_{i j}$ represents a shortdistance partonic scattering; and $\otimes$ indicates an integration over value of $x$ or $x^{\prime}$, accessible by the scattering cross section. By measuring hadronic cross sections, with perturbatively calculated partonic hard parts $d \hat{\sigma}_{i j}$, PDFs have

Published by the American Physical Society under the terms of the Creative Commons Attribution 4.0 International license. Further distribution of this work must maintain attribution to the author(s) and the published article's title, journal citation, and DOI. Funded by SCOAP. been extracted from the world data at state-of-the-art nextto-next-to-leading order (NNLO) accuracy [1].

With the steep falling nature of PDFs as $x \rightarrow 1$ and the convolution in Eq. (1), the uncertainty of extracted PDFs at large $x$ is so significant that it limits our confidence to push the search for signals of new physics to larger invariant mass. With the nonperturbative nature of PDFs, it is natural to ask if we can calculate PDFs directly in lattice QCD (LQCD). The short answer is no since the operators defining PDFs are time-dependent and LQCD is formulated in Euclidean space-time. Recently, stimulated by the quasi-PDFs approach [3] (it was later formulated in a largemomentum effective field theory [4,5]), extraction of PDFs from lattice QCD calculation has drawn a lot of attention and many new ideas have appeared, including the pseudoPDFs [6], current-current correlators in momentum space [7], and current-current correlators in position space [8]. See also some earlier related approaches [9-14].

As proposed by two of us in Refs. [8,15], PDFs can be extracted from any good LQCD observables, which are referred to as "Lattice Cross Sections" (LCSs), that are calculable in LQCD and factorizable into PDFs with perturbatively calculable matching coefficients,

$$
\begin{aligned}
\sigma_{n / h}\left(\omega, \xi^{2}\right) \equiv & \left\langle h(p)\left|T\left\{\mathcal{O}_{n}(\xi)\right\}\right| h(p)\right\rangle \\
= & \sum_{i} f_{i / h}\left(x, \mu^{2}\right) \otimes K_{n / i}\left(x \omega, \xi^{2}, \mu^{2}\right) \\
& +O\left(\xi^{2} \Lambda_{\mathrm{QCD}}^{2}\right),
\end{aligned}
$$


where $\xi$ with $\xi^{2} \neq 0$ represents the size of nonlocal operator $\mathcal{O}_{n}(\xi)$ of type $n$, controlling the short-distance physics of the factorization, $\omega \equiv p \cdot \xi$ (often referred as Ioffe time), and $K_{n / i}$ are perturbative matching coefficients. The PDFs in Eqs. (1) and (2) are the same, and can be extracted by QCD global fits of data generated by LQCD calculation of $\sigma_{n / h}\left(\omega, \xi^{2}\right)$ with various operator type $n$, together with the world data on various high energy scattering cross sections $[1,8,15,16]$.

One key difference between Eqs. (1) and (2) is that $\sigma_{n / h}$ in Eq. (2) is not an experimentally measured physical cross section. The corresponding operator $\mathcal{O}_{n}(\xi)$, which can be a two-quark correlation operator that defines quasi-PDFs [3], current-current correlators [8], or any others that satisfy the aforementioned properties, might require additional ultraviolet (UV) renormalization beyond using renormalized fields. This additional UV renormalization impacts the calculation and stability of the perturbative matching coefficients $K_{n / i}$ for LQCD observables. Although extraction of PDFs from LQCD calculations has made tremendous progress in recent years [17-55], the state-ofthe-art calculation of short-distance matching coefficients is still limited to the next-to-leading order (NLO) in almost all existing approaches [30-37], which is partially limited by this additional renormalization and our ability to do perturbative calculation in coordinate space. In this Letter, we derive for the first time the NNLO nonsinglet matching coefficients in dimensional regularization, allowing us to extract PDFs from LQCD calculations at the same rigor as those extracted from experimental data, as well as addressing concerns that the factorization might be invalidated at NNLO [56].

Quark correlation functions.-We focus on the following unpolarized gauge invariant quark correlation operator [3]:

$$
\mathcal{O}_{q}^{\nu, b}\left(\xi, \mu^{2}, \delta\right)=\left.\bar{\psi}_{q}(\xi) \gamma^{\nu} \Phi^{(f)}(\{\xi, 0\}) \psi_{q}(0)\right|_{\mu^{2}, \delta},
$$

which is made of renormalized fields with a path ordered gauge link $\mathrm{in}_{1}$ the fundamental representation, $\Phi^{(f)}(\xi, 0)=\mathcal{P} e^{-i g_{s} \int_{0}^{1} \xi \cdot A^{(f)}\left(r^{\xi}\right) d r}$. Because this composite quark correlation operator is UV divergent, a UV regulator $\delta$ is needed, which may represent lattice spacing $a$ in lattice QCD calculations, or represent $\epsilon \equiv(4-d) / 2$ in dimensional regularization (DR) of continuum calculations. $\mu$ is a dimensional scale accompanied by the UV regulator, which is different from the factorization scale in Eq. (2), while one could choose them to be equal numerically. This UV divergence is multiplicatively renormalizable [24-26], as

$$
\mathcal{O}_{q}^{\nu, \mathrm{RS}}(\xi)=\mathcal{O}_{q}^{\nu, b}\left(\xi, \mu^{2}, \delta\right) / Z^{\mathrm{RS}}\left(\xi^{2}, \mu^{2}, \delta\right)
$$

where superscript RS indicates a renormalization scheme and $Z^{\mathrm{RS}}\left(\xi^{2}, \mu^{2}, \delta\right)$ is the multiplicative renormalization constant. For regularization-invariant renormalization conditions, the renormalized $\mathcal{O}_{q}^{\nu, \mathrm{RS}}$ are independent of $\delta$ and $\mu^{2}$.

Quark correlation functions (QCFs) are defined as hadronic matrix elements of $\mathcal{O}_{q}^{\nu, \mathrm{RS}}(\xi)$

$$
F_{q / h}^{\nu, \mathrm{RS}}\left(\omega, \xi^{2}\right)=\left\langle h(p)\left|\mathcal{O}_{q}^{\nu, \mathrm{RS}}(\xi)\right| h(p)\right\rangle,
$$

which is independent of the regularization scheme and scale, like physical cross sections. With $\xi^{0}=0$ and $\xi^{2} \Lambda_{\mathrm{QCD}}^{2} \ll 1, F_{q / h}^{\nu, \mathrm{RS}}\left(\omega, \xi^{2}\right)$ are expected to be calculable in LQCD and proved to be factorizable into PDFs $[8,15,35]$. Their Fourier transform over $d \omega$ with fixed $p$ leads to the quasi-PDFs; and with fixed $\xi$ is proportional to pseudo-PDFs [8]. In this Letter, we focus on flavor nonsinglet combinations of QCFs, and have the corresponding factorization formula in continuum as [8]

$$
\begin{aligned}
F_{q_{i k} / h}^{\nu, \mathrm{RS}}\left(\omega, \xi^{2}\right)= & \frac{1}{R^{\mathrm{RS}}\left(\xi^{2}, \mu^{2}\right)} \int_{-1}^{1} \frac{d x}{x} f_{q_{i k} / h}\left(x, \mu^{2}\right) \\
& \times K^{\nu}\left(x \omega, \xi^{2}, \mu^{2}\right)+O\left(\xi^{2} \Lambda_{\mathrm{QCD}}^{2}\right),
\end{aligned}
$$

where $R^{\mathrm{RS}}\left(\xi^{2}, \mu^{2}\right) \equiv Z^{\mathrm{RS}}\left(\xi^{2}, \mu^{2}, \epsilon\right) / Z^{\overline{\mathrm{MS}}}\left(\xi^{2}, \mu^{2}, \epsilon\right)$ is a finite renormalization factor that transforms any "preferred" regularization-invariant RS scheme to the conventional $\overline{\mathrm{MS}}$ scheme, $K^{\nu}$ are perturbative matching coefficients in $\overline{\mathrm{MS}}$ scheme, and $q_{i k} \equiv q_{i}-q_{k}$ means

$$
\begin{gathered}
f_{q_{i k} / h}\left(x, \mu^{2}\right) \equiv f_{q_{i} / h}\left(x, \mu^{2}\right)-f_{q_{k} / h}\left(x, \mu^{2}\right), \\
F_{q_{i k} / h}^{\nu, \mathrm{RS}}\left(\omega, \xi^{2}\right) \equiv F_{q_{i} / h}^{\nu, \mathrm{RS}}\left(\omega, \xi^{2}\right)-F_{q_{k} / h}^{\nu, \mathrm{RS}}\left(\omega, \xi^{2}\right),
\end{gathered}
$$

where $q_{i}, q_{k}=u, d, s$ are quark flavors. To extract the nonsinglet distribution $f_{q_{i k} / h}$ from LQCD calculations of $F_{q_{i k} / h}^{\nu, \mathrm{RS}}$ to the NNLO accuracy, we have to perturbatively calculate $R^{\mathrm{RS}}$ and $K^{\nu}$ to the power of $\alpha_{s}^{2}$. The factorization formula in Eq. (6) is also valid for valencequark correlation functions by replacing $q_{i j}$ and $K^{\nu}\left(x \omega, \xi^{2}, \mu^{2}\right) \quad$ with $q_{v} \equiv q-\bar{q}$ and $K_{v}^{\nu}\left(x \omega, \xi^{2}, \mu^{2}\right) \equiv$ $K^{\nu}\left(x \omega, \xi^{2}, \mu^{2}\right)-K^{\nu}\left(-x \omega, \xi^{2}, \mu^{2}\right)$, respectively.

Renormalization constant.-The renormalization constant $Z^{\mathrm{RS}}$ introduced in Eq. (4) is determined by the short-distance property of the quark correlation operator in Eq. (3) and should not depend on the hadronic state used to define the QCFs of this operator. Because of its multiplicative renormalizability, the matrix element of $\mathcal{O}_{q}^{\nu, b}$ in Eq. (3) with any state could define an allowable renormalization scheme,

$$
Z^{\mathrm{RS}}\left(\xi^{2}, \mu^{2}, \delta\right)=\frac{\left\langle\mathrm{RS}\left|\hat{n} \cdot \mathcal{O}_{q}^{b}\left(\xi, \mu^{2}, \delta\right)\right| \mathrm{RS}\right\rangle}{\left\langle\mathrm{RS}\left|\hat{n} \cdot \mathcal{O}_{q}^{b}\left(\xi, \mu^{2}, \delta\right)\right| \mathrm{RS}\right\rangle^{(0)}},
$$

where $\hat{n}$ is any vector keeping the denominator nonvanishing and the superscript "( 0$)$ " indicates that the matrix element is evaluated to the leading order (LO) in perturbation theory. 
A different choice of the state $|\mathrm{RS}\rangle$ corresponds to a different renormalization scheme. For example, an off-shell quark state with a specific momentum was used in defining the regularization independent momentum subtraction scheme (RI-MOM) or its modified version $\mathrm{RI}^{\prime}-\mathrm{MOM}$ [27-31]; a hadron state with zero momentum was used in calculations of pseudo-PDFs [36] [the matrix element in this case cannot be perturbatively calculated and one may choose the denominator in Eq. (9) as 1]; and the vacuum state was introduced in Ref. [57].

In the following, we define the renormalization constant with the vacuum state $|\Omega\rangle$ and denote $\mathrm{RS}=$ vac. By calculating the vacuum expectation value to NNLO, we demonstrate that without an identified external momentum, the renormalization constant $Z^{\mathrm{vac}}$ is completely free of infrared (IR) and collinear (CO) singularity and its UV divergence is regularized by DR, from which we obtain $Z^{\mathrm{MS}}\left(\xi^{2}, \mu^{2}, \epsilon\right)$ and $R^{\mathrm{vac}}\left(\xi^{2}, \mu^{2}\right)$ at NNLO level.

In the top row of Fig. 1, we show representative LO (a), NLO (b) and NNLO (c) Feynman diagrams for the vacuum expectation $\left\langle\Omega\left|\hat{n} \cdot \mathcal{O}_{q}^{b}\left(\xi, \mu^{2}, \delta\right)\right| \Omega\right\rangle$. The diagram (a) in Fig. 1 determines the normalization of $Z^{\mathrm{vac}}$,

$\left\langle\Omega\left|\hat{n} \cdot \mathcal{O}_{q}^{\mathrm{b}}\right| \Omega\right\rangle^{(0)}=2 N_{c} \mu^{4-d} \pi^{-d / 2} \Gamma(d / 2)|\xi|^{-d} \hat{n} \cdot \xi$,

where $|\xi|^{2} \equiv-\xi^{2}$, and the result agrees with Ref. [57].

The Fig. 1(b) is a representative Feynman diagram contributing to NLO $Z^{\mathrm{vac}}$,

$$
\begin{aligned}
& M_{b}=g_{s}^{2} N_{c} C_{F} \mu^{8-2 d} \int_{0}^{1} d r \int \frac{d^{d} l_{1} d^{d} l_{2}}{(2 \pi)^{2 d}} e^{i l_{1} \cdot \xi+i r l_{2} \cdot \xi} \\
& \times \frac{\operatorname{Tr}\left[\left(l_{1}+l_{2}\right) \xi l_{1} \hat{n}\right]}{\left(l_{1}^{2}+i 0^{+}\right)\left(l_{2}^{2}+i 0^{+}\right)\left(\left(l_{1}+l_{2}\right)^{2}+i 0^{+}\right)},
\end{aligned}
$$

where we assume without loss of generality that $z$-component $\xi_{z}$ is only the nonzero component of $\xi$, and $\hat{n}$ satisfies $\hat{n}$.

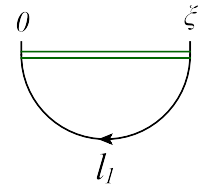

(a)

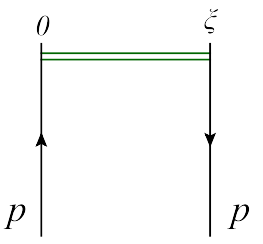

( $\left.a^{\prime}\right)$

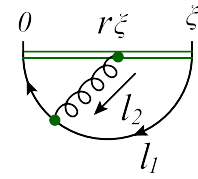

(b)

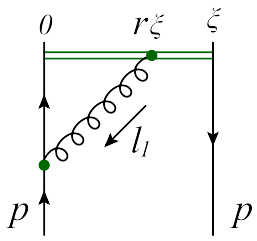

(b')

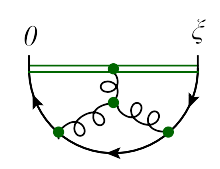

(c)

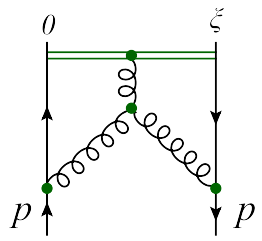

$\left(c^{\prime}\right)$
FIG. 1. Representative LO (a), NLO (b) and NNLO (c) Feynman diagrams for the vacuum expectation value of the quark correlation operator (top row), and for the nonsinglet quark matrix elements of the same operator (bottom row). $l \equiv l_{z}$ for any vector $l$. We find that it is convenient to carry out the integration in Eq. (11) by Fourier transforming the $\xi_{z}$ into $q_{z}$ in momentum space as $\mathcal{F}\left[M_{b}\right] \equiv \int d \xi_{z} e^{-i \xi_{z} q_{z}} M_{b}$ to eliminate the exponential factor by using

$$
\begin{aligned}
& \int d \xi_{z} e^{-i \xi_{z} q_{z}} \xi_{z} \int_{0}^{1} d r e^{-i l_{1 z} \xi_{z}-i r l_{2 z} \xi_{z}}, \\
& =2 i \operatorname{Im}\left(\frac{1}{\left(q_{z}+l_{1 z}+l_{2 z}+i 0^{+}\right)\left(q_{z}+l_{1 z}+i 0^{+}\right)}\right),
\end{aligned}
$$

where $2 \pi \delta(x)=-2 \operatorname{Im}\left[1 /\left(x+i 0^{+}\right)\right]$is used. The Fourier transformation also ensures that only the imaginary part of gauge-link-related propagators are involved, which led to the similar effect of the optical theorem. Our matrix element is defined with a gauge link in coordinate space, which is effectively equal to the sum over diagrams with a cut gauge link in momentum space. It is the summation of cuts of the gauge link that forces the appearance of the imaginary part of the "forward scattering amplitude." The obtained loop integrals in momentum space can be reduced to a linear combination of a small set of integrals, called master integrals (MIs), by using integration-by-parts relations (IBPs) $[58,59]$. We use the package FIRE5 [60] to do this reduction, which results in

$$
\begin{aligned}
\mathcal{F}\left[M_{b}\right]= & i g_{s}^{2} N_{c} C_{F} \mu^{8-2 d} \frac{2(d-2)}{d-4} \\
& \times\left[I_{1}-\frac{2(2 d-5)(3 d-10)}{(d-3)(d-4)} q_{z}^{-1} I_{2}\right],
\end{aligned}
$$

with two vacuum MIs defined as

$$
\begin{aligned}
I_{1}= & \int \frac{d^{d} l_{1} d^{d} l_{2}}{(2 \pi)^{2 d}} \frac{1}{\left(l_{1}^{2}+i 0^{+}\right)\left(l_{2}^{2}+i 0^{+}\right)} \\
& \times 2 \operatorname{Im}\left(\frac{1}{\left(q_{z}+l_{1 z}+i 0^{+}\right)\left(q_{z}+l_{2 z}+i 0^{+}\right)}\right), \\
I_{2}= & \int \frac{d^{d} l_{1} d^{d} l_{2}}{(2 \pi)^{2 d}} \frac{1}{\left(l_{1}^{2}+i 0^{+}\right)\left(l_{2}^{2}+i 0^{+}\right)} \\
& \times 2 \operatorname{Im}\left(\frac{1}{q_{z}+l_{1 z}+l_{2 z}+i 0^{+}}\right) .
\end{aligned}
$$

To carry out these single-scale vacuum MIs, we use the method presented in Ref. [61] by setting up and solving the dimensional recurrence relations and obtain

$I_{1}=\frac{\pi^{-d}}{8} \sin (d \pi) \Gamma(d / 2-1)^{2} \Gamma(3-d)^{2}\left|q_{z}\right|^{2 d-9} q_{z}^{3}$,

$I_{2}=\frac{\pi^{-d}}{8} \sin (d \pi) \Gamma(d / 2-1)^{2} \Gamma(5-2 d)\left|q_{z}\right|^{2 d-9} q_{z}^{4}$.

We then Fourier transform inversely from $q_{z}$ dependence into $\xi_{z}$ dependence to derive the result of $M_{b}$ in DR. Other 
two-loop diagrams, including UV counterterm diagrams, can be calculated similarly.

All three-loop diagrams like diagram (c) in Fig. 1 can also be calculated similarly as the diagram (b) described above. The only difference is that analytical expression of vacuum MIs cannot be obtained by solving dimensional recurrence relations directly. Instead, we calculate the vacuum MIs to high accuracy by using dimensional recurrence relations and then obtain exact results by using the PSLQ algorithm [62]. We check the correctness of our exact results numerically with at least $10^{3}$ digits.

By adding all diagrams and UV counterterms together, the remaining divergences should be removed by operator renormalization. With a $\overline{\mathrm{MS}}$ subtraction scheme, we obtain $Z^{\overline{\mathrm{MS}}}\left(\xi^{2}, \mu^{2}, \epsilon\right)$ and $R^{\mathrm{vac}}\left(\xi^{2}, \mu^{2}\right)$ at NNLO level, with analytical expressions given in Supplemental Material [74].

Matching coefficients.-By choosing the $\overline{\mathrm{MS}}$ scheme for QCFs, we have the same factorization in Eq. (6) with $R^{\mathrm{RS}}=1$, which leads to a $\mu$ dependence on the left hand of the equation. To calculate the matching coefficients $K^{\nu}$, we replace the hadron $h$ in Eq. (6) by a quark state and expand both sides perturbatively,

$$
\begin{aligned}
F_{q_{i k} / q_{i}}^{\nu(n)}\left(\omega, \xi^{2}, \mu^{2}\right)= & \sum_{m=0}^{n} \int_{-1}^{1} \frac{d x}{x} f_{q_{i k} / q_{i}}^{(m)}\left(x, \mu^{2}\right) \\
& \times K^{\nu(n-m)}\left(x \omega, \xi^{2}, \mu^{2}\right),
\end{aligned}
$$

with $n, m=0,1,2$ indicating the power in $\alpha_{s}$. While the partonic $f_{q_{i k}}^{(n)}$ with $n=0,1,2$ in the $\overline{\mathrm{MS}}$ factorization scheme are known [63], we have to calculate the partonic version of $F_{q_{i k} / q_{i}}^{\nu(n)}$ in the $\overline{\mathrm{MS}}$ scheme perturbatively to $n=0$, 1, 2 to derive the NNLO matching coefficient $K^{\nu(n)}$.

Some representative Feynman diagrams for $F_{q_{i k} / q_{i}}^{\nu(n)}$ are shown in Figs. 1(a'), 1(b'), and 1(c'). The diagram Fig. 1(a') gives the tree level result

$$
F_{q_{i k} / q_{i}}^{\nu(0)}=-2 i p^{\nu} e^{i \omega}
$$

To calculate $F_{q_{i k} / q_{i}}^{\nu}\left(\omega, \xi^{2}, \mu^{2}\right)$ at high orders, we again use transformation as in Eq. (12) to remove the exponential by going to momentum space, and then reduce the loop integrals to MIs by using IBPs. For example, at NLO we have two MIs:

$I_{1}^{(1)}=\int \frac{d^{d} l_{1}}{(2 \pi)^{d}} \frac{1}{l_{1}^{2}+i 0^{+}} 2 \operatorname{Im}\left(\frac{1}{q_{z}+l_{1 z}+i 0^{+}}\right)$,

$I_{2}^{(1)}=\int \frac{d^{d} l_{1}}{(2 \pi)^{d}} \frac{1}{l_{1}^{2}+i 0^{+}} 2 \operatorname{Im}\left(\frac{1}{q_{z}+l_{1 z}+p_{z}+i 0^{+}}\right)$

and at NNLO we have 21 MIs. The MIs generated from $n$-loop diagrams for $F_{q_{v} / q}^{\nu(n)}$ are functions that satisfy

$$
I_{j}^{(n)}\left(y, p_{z} ; d\right)=\left|q_{z}\right|^{d_{n}} q_{z}^{d_{n j}} J_{j}^{(n)}(y ; d),
$$

where $y \equiv p_{z} / q_{z}, d_{n} \equiv-2 n \epsilon-1$, and $d_{n}+d_{n j}$ are the dimensions of MI $I_{j}^{(n)}$. These MIs can be derived by solving the differential equations [64]

$$
\partial_{y} J_{j}^{(n)}(y ; d)=\sum_{k} A_{j k}(y ; d) J_{k}^{(n)}(y ; d),
$$

with $J_{j}^{(n)}(0 ; d)$ serving as boundary conditions. By applying IBPs again, the integrals in boundary conditions can be decomposed into vacuum MIs at $n$-loop order, which have been calculated in the renormalization procedure. Therefore, $J_{j}^{(n)}$ can be expanded as a Taylor series of $y$ based on the differential equations in Eq. (20).

After carrying out MIs, we can Fourier transform back to position space and the $y$ dependence is changed to dependence on $\omega$. Analytical results can be obtained by fitting the Taylor series of $\omega$ with the proper ansatz [65] in terms of harmonic polylogarithms [66-68]. By adding contributions from all diagrams and then multiplying it by UV renormalization factor $Z_{\overline{\mathrm{MS}}}^{-\frac{1}{2}}$, we obtain perturbative results of $F_{q_{i k} / q_{i}}^{\nu(n)}\left(\omega, \xi^{2}, \mu^{2}\right)$ with $n=1,2$. We then obtain $\overline{\mathrm{MS}}$ matching coefficients $K^{\nu(n)}\left(x \omega, \xi^{2}, \mu^{2}\right)$ using Eq. (16). As expected, all divergences are canceled and final results of $K^{\nu(n)}$ are finite. It verifies the proof of the factorization theorem [15] up to two-loop order. Our one-loop results $K^{\nu(1)}$ agree with previous calculations [33-35]; terms proportional to $n_{f}$ in two-loop results have been calculated in Ref. [57] using the quark mass regulator; while other two-loop results are new. By performing Fourier transformation, we also obtain analytical matching coefficients for pseudo-PDFs and quasi-PDFs. All analytical results are given in the Supplemental Material.

Using Eq. (6), one can obtain NNLO matching coefficients in other RSs by calculating the corresponding $R^{\mathrm{RS}}$.

Numerical results. - With our calculated matching coefficients at LO, NLO, and NNLO and the factorization formula in Eq. (6), we can predict valence-quark correlation functions by using existing PDFs extracted from experimental data, and test them by LQCD calculations. In Fig. 2, we present $(i / 4 \omega) \xi \cdot F_{q_{v} / h}^{\mathrm{vac}}\left(\omega, \xi^{2}\right)$ as a function of $\omega$ with fixed $1 /|\xi|=2 \mathrm{GeV}$ or as a function of $1 /|\xi|$ with fixed $\omega=10$. We used CT18NNLO PDFs [69], and set $\mu=2 c /|\xi|$ to minimize logarithms encountered in perturbative calculation. We chose $c=1$ for the predicted curves, and varied $c=1 / 2$ to 2 for the bands to estimate theoretical uncertainties due to ambiguity of scale choice. Our numerical predictions show a great improvement in perturbative uncertainty when NNLO matching is used, especially, for the region where $1 /|\xi|$ is small and more lattice data are available [16]. The NNLO results can reduce theoretical uncertainty by more than a factor of 3 comparing to NLO results. 

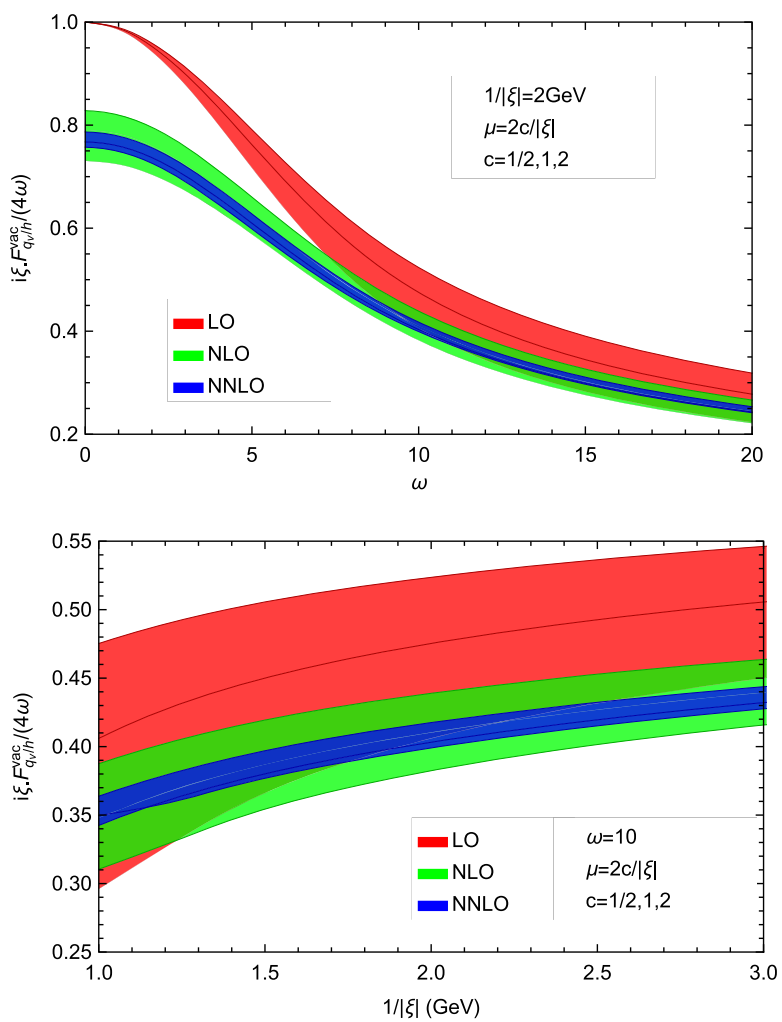

FIG. 2. Numerical predictions for valence-quark correlation functions with LO, NLO, and NNLO matching coefficients and CT18NNLO PDFs.

Summary.-Properly renormalized quark correlation functions in position space, if $\xi^{2} \Lambda_{\mathrm{QCD}}^{2}$ is sufficiently small, are good LQCD observables that are calculable in LQCD and factorizable to PDFs $[8,15,35]$. We discussed the ambiguity and scheme dependence of the multiplicative renormalization constant $Z^{\mathrm{RS}}$, and demonstrated that $Z^{\mathrm{RS}}$ defined with the vacuum state is advantageous for carrying out perturbative calculations of the matching coefficients, especially, at high order in $\alpha_{s}$. For the first time, we derived complete NNLO flavor nonsinglet coefficient functions for QCFs, and predicted valence-quark correction functions in Fig. 2 by using existing PDFs and our matching coefficients. We clearly demonstrated the importance of NNLO matching coefficients for reducing the perturbative uncertainty in our factorization approach. Comparing our predictions with LQCD data will provide the first test of compatibility between LQCD calculations and high energy experimental measurements in terms of QCD factorization at the NNLO accuracy [16].

Our definition of QCFs and method of calculations can be easily generalized to gluon correlation functions (GCFs). With multiple "good" LQCD observables, including the QCFs and GCFs, as well as the currentcurrent correlation functions (better UV behavior) [8], and our ability to calculate NNLO matching coefficients, the extraction of PDFs from LQCD calculations in terms of
QCD factorization approach can be in fact at the same rigor as how PDFs have been extracted from experimental data. In addition to the complementary revenue for extracting PDFs or other partonic structures of hadrons, LQCD calculation provides a tremendous potential to extract the partonic structure of hadrons that could be difficult to do scattering experiments with.

We thank L. Leskovec, R. Sufian, and Y.-B. Yang for useful discussions. The work of Z.-Y.L. and Y.-Q. M. is supported by the National Natural Science Foundation of China (Grants No. 11875071, No. 11975029) and the Highperformance Computing Platform of Peking University, and J.-W. Q. is supported by the U.S. Department of Energy Contract No. DE-AC05-06OR23177, under which Jefferson Science Associates, LLC, manages and operates Jefferson Lab.

Note added.-Recently, some related preprints appeared [70-72]. In Ref. [70] the authors obtained NNLO results for $Z^{\overline{\mathrm{MS}}}$ and $R^{\mathrm{vac}}$, which exactly agree with our results. In Refs. [71,72] the authors obtained matching coefficients for flavor nondiagonal quark to quark channel that starts from two-loop order. Reference [73], where flavor nonsinglet matching coefficients for quasi-PDF are also calculated to NNLO, includes results that are in agreement with ours.

*lizhengyang@pku.edu.cn

yqma@pku.edu.cn \#jqiu@jlab.org

[1] H.-W. Lin et al., Parton distributions and lattice QCD calculations: Toward 3D structure, arXiv:2006.08636.

[2] J. C. Collins, D. E. Soper, and G. Sterman, Factorization of hard processes in QCD, Adv. Ser. Dir. High Energy Phys. 5, 1 (1988).

[3] X. Ji, Parton Physics on a Euclidean Lattice, Phys. Rev. Lett. 110, 262002 (2013).

[4] X. Ji, Parton Physics from large-momentum effective field theory, Sci. China Phys. Mech. Astron. 57, 1407 (2014).

[5] X. Ji, Y.-S. Liu, Y. Liu, J.-H. Zhang, and Y. Zhao, Largemomentum effective theory, arXiv:2004.03543.

[6] A. Radyushkin, Quasi-parton distribution functions, momentum distributions, and pseudo-parton distribution functions, Phys. Rev. D 96, 034025 (2017).

[7] A. J. Chambers, R. Horsley, Y. Nakamura, H. Perlt, P. E. L. Rakow, G. Schierholz, A. Schiller, K. Somfleth, R. D. Young, and J. M. Zanotti, Nucleon Structure Functions from Operator Product Expansion on the Lattice, Phys. Rev. Lett. 118, 242001 (2017).

[8] Y.-Q. Ma and J.-W. Qiu, Exploring Partonic Structure of Hadrons Using ab initio Lattice QCD Calculations, Phys. Rev. Lett. 120, 022003 (2018).

[9] K.-F. Liu and S.-J. Dong, Origin of Difference between Anti-d and Anti-u Partons in the Nucleon, Phys. Rev. Lett. 72, 1790 (1994).

[10] K.-F. Liu, Parton degrees of freedom from the path integral formalism, Phys. Rev. D 62, 074501 (2000). 
[11] K.-F. Liu, Parton distribution function from the hadronic tensor on the lattice, Proc. Sci., LATTICE2015 (2016) 115.

[12] U. Aglietti, M. Ciuchini, G. Corbo, E. Franco, G. Martinelli, and L. Silvestrini, Model independent determination of the light cone wave functions for exclusive processes, Phys. Lett. B 441, 371 (1998).

[13] A. Abada, P. Boucaud, G. Herdoiza, J. P. Leroy, J. Micheli, O. Pene, and J. Rodriguez-Quintero, Preliminaries on a lattice analysis of the pion light cone wave function: A Partonic signal?, Phys. Rev. D 64, 074511 (2001).

[14] V. Braun and D. Mueller, Exclusive processes in position space and the pion distribution amplitude, Eur. Phys. J. C 55, 349 (2008).

[15] Y.-Q. Ma and J.-W. Qiu, Extracting parton distribution functions from lattice QCD calculations, Phys. Rev. D 98, 074021 (2018).

[16] J. Bringewatt, N. Sato, W. Melnitchouk, J.-W. Qiu, F. Steffens, and M. Constantinou, Confronting lattice parton distributions with global QCD analysis, Phys. Rev. D 103, 016003 (2021)..

[17] T. Ishikawa, Y.-Q. Ma, J.-W. Qiu, and S. Yoshida, Practical quasi parton distribution functions, arXiv:1609.02018.

[18] J.-W. Chen, X. Ji, and J.-H. Zhang, Improved quasi parton distribution through Wilson line renormalization, Nucl. Phys. B915, 1 (2017).

[19] C. Monahan and K. Orginos, Quasi parton distributions and the gradient flow, J. High Energy Phys. 03 (2017) 116.

[20] R. A. Briceño, M. T. Hansen, and C. J. Monahan, Role of the Euclidean signature in lattice calculations of quasidistributions and other nonlocal matrix elements, Phys. Rev. D 96, 014502 (2017).

[21] X. Xiong, T. Luu, and U.-G. Meißner, Quasi-parton distribution function in lattice perturbation theory, arXiv:1705.00246.

[22] Z.-Y. Li, Y.-Q. Ma, and J.-W. Qiu, Multiplicative Renormalizability of Operators defining Quasiparton Distributions, Phys. Rev. Lett. 122, 062002 (2019).

[23] J.-H. Zhang, X. Ji, A. Schäfer, W. Wang, and S. Zhao, Accessing Gluon Parton Distributions in Large Momentum Effective Theory, Phys. Rev. Lett. 122, 142001 (2019).

[24] X. Ji, J.-H. Zhang, and Y. Zhao, Renormalization in Large Momentum Effective Theory of Parton Physics, Phys. Rev. Lett. 120, 112001 (2018).

[25] T. Ishikawa, Y.-Q. Ma, J.-W. Qiu, and S. Yoshida, Renormalizability of quasiparton distribution functions, Phys. Rev. D 96, 094019 (2017).

[26] J. Green, K. Jansen, and F. Steffens, Nonperturbative Renormalization of Nonlocal Quark Bilinears for QuasiPDFs on the Lattice Using an Auxiliary Field, Phys. Rev. Lett. 121, 022004 (2018).

[27] M. Constantinou and H. Panagopoulos, Perturbative renormalization of quasi-parton distribution functions, Phys. Rev. D 96, 054506 (2017).

[28] C. Alexandrou, K. Cichy, M. Constantinou, K. Hadjiyiannakou, K. Jansen, H. Panagopoulos, and F. Steffens, A complete non-perturbative renormalization prescription for quasi-PDFs, Nucl. Phys. B923, 394 (2017).

[29] J.-W. Chen, T. Ishikawa, L. Jin, H.-W. Lin, Y.-B. Yang, J.-H. Zhang, and Y. Zhao, Parton distribution function with non-perturbative renormalization from lattice QCD, Phys. Rev. D 97, 014505 (2018).
[30] I. W. Stewart and Y. Zhao, Matching the quasiparton distribution in a momentum subtraction scheme, Phys. Rev. D 97, 054512 (2018).

[31] W. Wang, J.-H. Zhang, S. Zhao, and R. Zhu, Complete matching for quasidistribution functions in large momentum effective theory, Phys. Rev. D 100, 074509 (2019).

[32] X. Xiong, X. Ji, J.-H. Zhang, and Y. Zhao, One-loop matching for parton distributions: Non-singlet case, Phys. Rev. D 90, 014051 (2014).

[33] X. Ji, J.-H. Zhang, and Y. Zhao, More on large-momentum effective theory approach to parton physics, Nucl. Phys. B924, 366 (2017).

[34] A. Radyushkin, Quark pseudodistributions at short distances, Phys. Lett. B 781, 433 (2018).

[35] T. Izubuchi, X. Ji, L. Jin, I. W. Stewart, and Y. Zhao, Factorization theorem relating Euclidean and light-cone parton distributions, Phys. Rev. D 98, 056004 (2018).

[36] K. Orginos, A. Radyushkin, J. Karpie, and S. Zafeiropoulos, Lattice QCD exploration of parton pseudo-distribution functions, Phys. Rev. D 96, 094503 (2017).

[37] R. S. Sufian, C. Egerer, J. Karpie, R. G. Edwards, B. Joó, Y.-Q. Ma, K. Orginos, J.-W. Qiu, and D. G. Richards, Pion valence quark distribution from current-current correlation in lattice QCD, Phys. Rev. D 102, 054508 (2020).

[38] H.-W. Lin, J.-W. Chen, S. D. Cohen, and X. Ji, Flavor Structure of the nucleon sea from lattice QCD, Phys. Rev. D 91, 054510 (2015).

[39] C. Alexandrou, K. Cichy, V. Drach, E. Garcia-Ramos, K. Hadjiyiannakou, K. Jansen, F. Steffens, and C. Wiese, Lattice calculation of parton distributions, Phys. Rev. D 92, 014502 (2015).

[40] J.-W. Chen, S. D. Cohen, X. Ji, H.-W. Lin, and J.-H. Zhang, Nucleon helicity and transversity parton distributions from lattice QCD, Nucl. Phys. B911, 246 (2016).

[41] C. Alexandrou, K. Cichy, M. Constantinou, K. Hadjiyiannakou, K. Jansen, F. Steffens, and C. Wiese, Updated lattice results for parton distributions, Phys. Rev. D 96, 014513 (2017).

[42] J.-H. Zhang, J.-W. Chen, X. Ji, L. Jin, and H.-W. Lin, Pion distribution amplitude from lattice QCD, Phys. Rev. D 95 , 094514 (2017).

[43] C. Monahan, Smeared quasidistributions in perturbation theory, Phys. Rev. D 97, 054507 (2018).

[44] T. Ishikawa, L. Jin, H.-W. Lin, A. Schäfer, Y.-B. Yang, J.-H. Zhang, and Y. Zhao, Gaussian-weighted parton quasidistribution (Lattice Parton Physics Project $\left(\mathrm{LP}^{3}\right)$ ), Sci. Chin. Phys. Mech. Astron. 62, 991021 (2019).

[45] C. Alexandrou, K. Cichy, M. Constantinou, K. Jansen, A. Scapellato, and F. Steffens, Light-Cone Parton Distribution Functions from Lattice QCD, Phys. Rev. Lett. 121, 112001 (2018).

[46] J.-W. Chen, L. Jin, H.-W. Lin, Y.-S. Liu, Y.-B. Yang, J.-H. Zhang, and Y. Zhao, Lattice calculation of parton distribution function from LaMET at physical pion mass with large nucleon momentum, arXiv:1803.04393.

[47] J.-H. Zhang, J.-W. Chen, L. Jin, H.-W. Lin, A. Schäfer, and Y. Zhao, First direct lattice-QCD calculation of the $x$-dependence of the pion parton distribution function, Phys. Rev. D 100, 034505 (2019). 
[48] Y.-S. Liu, J. W. Chen, Y. K. Huo, L. Jin, M. Schlemmer, A. Schafer, P. Sun, W. Wang, Y. B. Yang, J. H. Zhang, Q. A. Zhang, K. Zhang, and Y. Zhao (Lattice Parton Collaboration), Unpolarized isovector quark distribution function from lattice QCD: A systematic analysis of renormalization and matching, Phys. Rev. D 101, 034020 (2020).

[49] G. S. Bali, V. M. Braun, B. Gläßle, M. Göckeler, M. Gruber, F. Hutzler, P. Korcyl, A. Schäfer, P. Wein, and J.-H. Zhang, Pion distribution amplitude from Euclidean correlation functions: Exploring universality and higher-twist effects, Phys. Rev. D 98, 094507 (2018).

[50] A. Radyushkin, Structure of parton quasi-distributions and their moments, Phys. Lett. B 788, 380 (2019).

[51] H.-W. Lin, J.-W. Chen, X. Ji, L. Jin, R. Li, Y.-S. Liu, Y.-B. Yang, J.-H. Zhang, and Y. Zhao, Proton Isovector Helicity Distribution on the Lattice at Physical Pion Mass, Phys. Rev. Lett. 121, 242003 (2018).

[52] J. Karpie, K. Orginos, and S. Zafeiropoulos, Moments of Ioffe time parton distribution functions from non-local matrix elements, J. High Energy Phys. 11 (2018) 178.

[53] B. Joo, J. Karpie, K. Orginos, A. V. Radyushkin, D. G. Richards, R. S. Sufian, and S. Zafeiropoulos, Pion valence structure from Ioffe-time parton pseudodistribution functions, Phys. Rev. D 100, 114512 (2019).

[54] Y. Chai et al., Parton distribution functions of $\Delta^{+}$on the lattice, Phys. Rev. D 102, 014508 (2020).

[55] H.-W. Lin, J.-W. Chen, Z. Fan, J.-H. Zhang, and R. Zhang, The valence-quark distribution of the kaon from lattice QCD, arXiv:2003.14128.

[56] H.-n. Li, Nondipolar Wilson links for quasiparton distribution functions, Phys. Rev. D 94, 074036 (2016).

[57] V. M. Braun, A. Vladimirov, and J.-H. Zhang, Power corrections and renormalons in parton quasidistributions, Phys. Rev. D 99, 014013 (2019).

[58] K. G. Chetyrkin and F. V. Tkachov, Integration by parts: The algorithm to calculate beta functions in 4 loops, Nucl. Phys. B192, 159 (1981).

[59] S. Laporta, High precision calculation of multiloop Feynman integrals by difference equations, Int. J. Mod. Phys. A 15, 5087 (2000).

[60] A. V. Smirnov, FIRE5: A C ++ implementation of Feynman Integral REduction, Comput. Phys. Commun. 189, 182 (2015).
[61] R. N. Lee, Calculating multiloop integrals using dimensional recurrence relation and $D$-analyticity, Nucl. Phys. B, Proc. Suppl. 205-206, 135 (2010).

[62] D. H. Bailey and D. J. Broadhurst, Parallel integer relation detection: Techniques and applications, Math. Comput. 70, 1719 (2001).

[63] G. Curci, W. Furmanski, and R. Petronzio, Evolution of parton densities beyond leading order: The nonsinglet case, Nucl. Phys. B175, 27 (1980).

[64] A. V. Kotikov, Differential equations method: New technique for massive Feynman diagrams calculation, Phys. Lett. B 254, 158 (1991).

[65] S. Moch, J. Vermaseren, and A. Vogt, The three loop splitting functions in QCD: The nonsinglet case, Nucl. Phys. B688, 101 (2004).

[66] A. B. Goncharov, Multiple polylogarithms, cyclotomy and modular complexes, Math. Res. Lett. 5, 497 (1998).

[67] E. Remiddi and J. Vermaseren, Harmonic polylogarithms, Int. J. Mod. Phys. A 15, 725 (2000).

[68] J. M. Borwein, D. M. Bradley, D. J. Broadhurst, and P. Lisonek, Special values of multiple polylogarithms, Trans. Am. Math. Soc. 353, 907 (2001).

[69] T.-J. Hou et al., New CTEQ global analysis of quantum chromodynamics with high-precision data from the LHC, , Phys. Rev. D 103, 014013 (2021).

[70] V. Braun, K. Chetyrkin, and B. Kniehl, Renormalization of parton quasi-distributions beyond the leading order: Spacelike vs. timelike, J. High Energy Phys. 07 (2020) 161.

[71] L.-B. Chen, W. Wang, and R. Zhu, Quasi parton distribution functions at NNLO: Flavor non-diagonal quark contributions, Phys. Rev. D 102, 011503 (2020).

[72] L.-B. Chen, W. Wang, and R. Zhu, Master Integrals for two-loop QCD corrections to Quasi PDFs, J. High Energy Phys. 10 (2020) 079.

[73] L.-B. Chen, W. Wang, and R. Zhu, following Letter, Next-to-next-to-Leading Order Corrections to Quark Quasi Parton Distribution Functions, Phys. Rev. Lett. 126, 072002 (2021).

[74] See Supplemental Material at http://link.aps.org/supplemental/ 10.1103/PhysRevLett.126.072001 for analytical results of renormalization factor for quark correlation operator and matching coefficients for quark correlation functions, quark pseudo-PDFs and quark quasi-PDFs up to NNLO. 